\title{
$\begin{array}{ll}\text { Research Square } & \text { Preprints are preliminary reports that have not undergone peer review. } \\ \text { They should not be considered conclusive, used to inform clinical practice, } \\ \text { or referenced by the media as validated information. }\end{array}$
}

\section{High Expression of PDZ-binding Kinase Is Correlated With Poor Prognosis and Immune Infiltrates in Hepatocellular Cacinoma}

\section{Wei Mu}

First Hospital of Shanxi Medical University https://orcid.org/0000-0001-9288-2917

Yaoli Xie

Shanxi Medical University

Jinhu Li

First Hospital of Shanxi Medical University

Runzhi Yan

First Hospital of Shanxi Medical University

Jingxian Zhang

Shanxi Provincial Peoples Hospital

Yu'e Liu

Shanxi Provincial Peoples Hospital

Yimin Fan ( $\square$ blueskyfan@126.com )

First Hospital of Shanxi Medical University

\section{Research Article}

Keywords: PDZ-binding kinase (PBK), Hepatocellular carcinoma (HCC), Tumor Infiltrating Immune Cells (TIICs), Bioinformatics analysis, Signaling pathways

Posted Date: September 14th, 2021

DOI: https://doi.org/10.21203/rs.3.rs-870796/v1

License: (a) (1) This work is licensed under a Creative Commons Attribution 4.0 International License. Read Full License 


\section{Abstract}

\section{Background}

PDZ-binding kinase (PBK) encodes a serine/threonine protein kinase related to the dual specific mitogenactivated protein kinase kinase (MAPKK) family. There is evidence that overexpression of this gene is associated with tumorigenesis. However, the role of PBK in hepatocellular carcinoma (HCC) remain unclear. Therefore, we evaluated the prognostic role of PBK and its correlation with immune infiltrates in Hepatocellular Carcinoma.

\section{Methods}

The expression of PBK in pan-cancers was studied by Onconmine and TIMER. The expression of PBK in $\mathrm{HCC}$ patients and its relationship with clinicopathological characteristics were analyzed using The Gene Expression Profiling Interactive Analysis (GEPIA), The human protein atlas database (HPA), The Cancer Genome Atlas (TCGA) and Gene Expression Omnibus (GEO) databases. Receiver operating characteristic (ROC) curve was used to determine the diagnostic value of PBK in HCC patients. The relationship between PBK and prognosis of HCC was performed by GEPIA and Kaplan Meier plotter web tool. The correlations between the clinical characteristics and overall survival were analyzed by Univariate Cox regression and Multivariate Cox hazards regression to identify possible prognostic factors for HCC patients. LinkedOmics was applied to investigate co-expression associated with PBK and to analyze Gene Ontology (GO) and Kyoto Encyclopedia of Genes and Genomes (KEGG) pathways. The network map of PBK and related genes is constructed by GeneMANIA. Finally, TIMER and TISIDB were used to analyze the correlations between PBK and tumor-infiltrating immune cells.

\section{Results}

PBK was highly expressed in many types of tumors, including hepatocellular carcinoma, and was significantly related to tumor stage $(P=0.0089)$, age $(P=0.0131)$ and race $(P=0.0024)$ of HCC patients. The receiver operating characteristic (ROC) curve analysis from 4 GEO databases showed that PBK is a predictive marker for HCC. Moreover, high expression of PBK correlated with poor prognosis. Multivariate Cox hazards regression showed that high expression of PBK may be an independent risk factor for overall survival in $\mathrm{HCC}$ patients $(\mathrm{HR}=1.566,95 \% \mathrm{Cl}=1.062-2.311, P=0.024)$. The Protein-protein interaction network showed that PBK significantly interacted with LRRC47, ARAF, LGALS9B, TTK, MELK and other essential genes. Furthermore, enrichment analysis showed that PBK and co-expressed genes participated in many biological processes, cell composition, molecular functions and pathways in HCC. Finally, the immune infiltration analysis by TIMER and TISIDB indicated that PBK expression tightly correlated with the infiltration of tumor-infiltrating lymphocytes (TILs), chemokines, and receptors.

\section{Conclusions}

High expression of PBK is significantly correlated with poor survival and immune infiltrates in hepatocellular carcinoma. Our study suggests that PBK can be used as a biomarker of poor prognosis 
and potential immune therapy target in hepatocellular carcinoma.

\section{Introduction}

Liver cancer is the sixth most common cancer and the fourth leading cause of cancer-related death in the world[1]. Hepatocellular carcinoma (HCC) accounts for about $75-85 \%$ of primary liver cancer and is a major health problem worldwide[2]. The causes of liver cancer occurrence and death are Hepatitis B virus (HBV) (33\%), alcohol (30\%), hepatitis C virus (HCV) $(21 \%)$ and other causes (16\%)[1]. In the early stage of $\mathrm{HCC}$, treatment includes liver resection, liver transplantation and ablation, and the prognosis is satisfactory. In the medium term, palliative locoregional treatment such as transcatheter arterial chemoembolization (TACE) remains the gold standard. However, most cases of HCC are diagnosed in the late stage. At that time, surgical treatment or TACE is no longer available and systematic treatment may be a better choice, but the prognosis is still poor[3].

With the development of DNA sequencing technology, precision medicine has made great progress, and many new therapeutic targets have been recognized. PDZ-binding kinase (PBK) is a member of the mitogen-activated protein kinase (MAPK) family, which acts on oncogenes as upstream kinases in some important signaling pathways[4]. PBK is highly expressed in testicular and fetal samples, but rarely found in other normal tissues[5]. Physiologically, through phosphorylation of various targets, it plays an active regulatory role in the proper separation of chromosomes and cytokinesis[6]. Current studies have shown that the increased expression of PBK is closely related to the occurrence and development of multiple tumors[7, 8], including lung cancer[9], ovarian carcinoma[10], oral cancer[11], prostate cancer[12], gastric carcinoma[13]. Therefore, the relationship between the expression of PBK and the prognosis of HCC, as well as the correlation between PBK and immune regulation is worth exploring.

In this study, we first analyzed the PBK expression and prognostic value in HCC by bioinformatics. Subsequently, we explored the association of PBK expression and immune infiltrating via TIMER and TISIDB databases. These findings will help understand the potential role of PBK in tumor immunology and its prognostic value.

\section{Materials And Methods}

\section{Data acquisition}

The RNA-seq data of HCC from The Cancer Genome Atlas (TCGA) was downloaded from the cBioPortal platform[14,15]. Clinicopathological information, including gender, age, tumor grade, race and survival data were also retrieved from the platform; patients who lacked complete information were excluded. We also downloaded the microarray dataset GSE84402, GSE76427, GSE62232 and GSE121248 from the Gene Expression Omnibus (GEO) database. In total, data relating to HCC in the TCGA related to 311 specimens; GSE84402 featured 14 samples of HCC tissue and 14 adjacent non-tumor specimens; GSE76427 featured 115 samples of HCC tissue and 52 adjacent non-tumor specimens; GSE62232 
featured 81 samples of HCC tissue and 10 adjacent non-tumor specimens; GSE121248 featured 70 samples of HCC tissue and 37 adjacent non-tumor specimens. Both mRNA expression profiles and clinical information are open-access and publicly available. Therefore, this study did not need approval from the Ethics Committee.

\section{The expression of PBK in pan-cancers}

To investigate the expression of PBK gene in pan-cancers, two online databases were used respectively -Oncomine and TIMER. There are 715 datasets and 86733 specimens collected by Oncomine web applications (https://www.oncomine.org/)[16]. These datasets come from public repositories, such as GEO, TCGA dataset, etc. In this study, we used Oncomine to analyze the expression of PBK in pan-cancers with P-value:0.05, the fold change:2, gene rank: top $10 \%$, data type: DNA+mRNA. TIMER (http://timer.comp-genomics.org/)[17,18] is a comprehensive resource for systematical analysis of immune infiltrates across diverse cancer types, which allows us to study the differential expression between tumor and adjacent normal tissues for PBK across all TCGA tumors by Gene_DE module. The statistical significance computed by the Wilcoxon test is annotated by the number of stars $(*$ : $p$-value < $0.05 ; * *$ : p-value $<0.01 ; * \star *$ : p-value $<0.001)$.

\section{Expression profile and diagnostic value of PBK in HCC and normal tissue}

HCCDB (http://lifeome.net/database/hccdb/)[19] is a database of hepatocellular carcinoma expression atlas, which consists of 15 public HCC expression datasets with up to about 4000 clinical samples. Gene Expression Profiling Interactive Analysis (GEPIA) (http://gepia.cancer-pku.cn/)[20] is a newly developed interactive web server for analyzing the RNA sequencing expression data of 9,736 tumors and 8,587 normal samples from the TCGA and the GTEx projects, which provides interactive functions, including differential expression analysis, mapping, correlation analysis, and survival analysis, etc. In this study, we used these online tools to analyze the expression of PBK in HCC and normal tissue. The human protein atlas database (HPA) (https://www.proteinatlas.org/)[21] provides tissue and cell distribution information of all 24000 kinds of human proteins, and provides free public query. We used this online database to explore the protein expression of PBK in HCC and normal tissues. We also downloaded PBK expression profiles from 4 independent GEO databases to validate the expression of PBK and to assess the diagnostic value of PBK by receiver operating characteristic (ROC) curves and the area under the curve (AUC) in HCC. Finally, the correlation analysis of PBK and clinicopathological features according to the TCGA-LIHC dataset were examined.

\section{Prognostic analysis from online database and TCGA-LIHC clinical information}

GEPIA can provide an association between PBK gene expression level and prognosis in patients with LIHC. The analysis indicators of GEPIA included Overall Survival and Disease-Free Survival, and the group cut-off is median. Kaplan Meier plotter (http://kmplot.com/analysis)[22] is a friendly website for online analysis of the impact of genes on the survival of cancer patients. It included $364 \mathrm{HCC}$ patients for Overall Survival (OS) analysis, 316 for Recurrence-Free Survival (RFS) analysis, 370 for Progression-Free 
Survival (PFS) analysis, and 362 for Disease-Specific Survival (DSS) analysis. In addition, A total of 311 HCC patients were included in the overall survival (OS) analysis from TCGA-LIHC database. Median expression of PBK of all samples was chosen as a cut-off to divide samples into the PBK-high $(n=155)$ group and PBK-low $(n=156)$ group. The Kaplan-Meier analysis was performed to investigate the prognosis of patients in the two groups. Univariate Cox regression and Multivariate Cox hazards regression analysis were performed to identify possible prognostic factors for HCC patients.

\section{GeneMANIA}

GeneMANIA (http://genemania.org/)[23] is an online site for discovering relevant genes and building visual networks of protein-protein interactions (PPI). The network map of PBK and related genes is constructed by GeneMANIA, which can also show the mode of interaction and functional analysis. In this map, each small circles represent different proteins in the network, the size of the circle represents the strength of the interaction, different colors of the attachment have different means of interaction.

\section{Linkedomics}

LinkedOmics (http://www.linkedomics.org/)[24] is an open and free online data analysis website, which contains multi omics data of all 32 TCGA cancer types[24]. We used the LinkFinder module of LinkeDomics website to find genes closely related to PBK in TCGA cohort, and displayed in the form of volcano and heat map. Besides, LinkInterpreter module of LinkedOmics was applied to conducted analyses of Gene Ontology (Biological Process, Cell Composition, Molecular Function) and Kyoto Encyclopedia of Genes and Genomes (KEGG) pathways through Gene Set Enrichment Analysis (GSEA).

\section{Tumor Immune Estimation Resource (TIMER) Database}

The version of TIMER2.0 provides immune infiltrates' abundances estimated by multiple immune deconvolution methods, and allows users to generate high-quality figures dynamically to explore tumor immunological, clinical and genomic features comprehensively[25]. Online analysis gene module was used to investigate the correlation between PBK expression and the abundance of CD4+T cells, CD8+T cells, B cells, macrophages, neutrophils and dendritic cells by correcting part of Spearman correlation with tumor purity. Then, we used outcome module to explore Kaplan-Meier curves for the corresponding immune infiltrates and HCC. Prognostic factors were assessed using a multivariable Cox proportional hazard model.

\section{Tumor-Immune System Interaction Database (TISIDB)}

TISIDB (http://cis.hku.hk/TISIDB/)[26] is an online web integrated repository portal for tumor immune system interaction. In this study, we performed TISIDB to determine the correlations between expression of PBK and abundance of subsets of tumor-infiltrating lymphocytes (TILs), chemokines, and receptors across human cancers.

\section{Statistical analysis}


All statistical analyses and graph were performed in SPSS21.0, GraphPad Prism 8 software and web resources. The measurement data are presented as mean \pm SD. Unpaired T-test and paired T-test were used to analyze the differential expression levels of PBK mRNA between the HCC tissues and the normal tissues from the GEO databases. ROC curve was performed to detect the diagnostic value of PBK in HCC. Kaplan-Meier and log-rank tests were conducted to assess the effect of PBK on survival. Univariate and multivariate analyses were performed using Cox proportional hazards regression model. Statistically significant differences were considered when $\mathrm{P}<0.05$.

\section{Results}

\section{Expression level of PBK in different types of human cancer}

To compare the differential expression of PBK gene in various tumors, the Oncomine database was used to analyze PBK mRNA levels. This analysis showed that when comparing cancer samples with normal samples, PBK gene is overexpressed in many tumors, including Liver Cancer but except Leukimia. The results also showed that PBK is overexpressed in 3 Liver Cancer datasets, but was not low-expressed in any of the Liver Cancer datasets (Fig. 1A).

To further evaluate PBK expression in human cancers, we validated PBK expression using RNA-seq data from multiple malignancies in TIMER. Compared to normal tissues, PBK expression was extremely higher in most common tumor tissues, such as Colon adenocarcinoma, Cholangiocarcinoma, Breast invasive carcinoma, Liver hepatocellular carcinoma and so on (Fig. 1B).

\section{High expression of PBK in HCC}

HCCDB is a public database of liver cancer. In this database, the expression of PBK in HCC tissues in 10 datasets was significantly higher than that in adjacent tissues or cirrhotic tissues, healthy tissues (Fig. 2A). Analysis from GEPIA shows that the expression level of PBK was significantly increased in HCC tissues (Fig. 2B).

HPA database is used for protein level detection, and the results showed that the expression of PBK in $\mathrm{HCC}$ was observed in the cytoplasmic/membranous nuclear. There was no expression of PBK in normal liver tissue, but significantly increased in HCC. Antibody used in the HPA database was HPA050656 (Fig. 2C).

\section{Validation from GEO database and the diagnostic value of PBK in HCC}

We used 4 independent GEO databases to verify PBK mRNA expression in HCC and matched normal tissues. Compared to normal tissues, PBK mRNA levels were upregulated in HCC tissues, based on GSE76427(Fig.3A), GSE121248(Fig.3B), GSE62232(Fig.3C) and GSE84402 (Fig.3D). The receiver operating characteristic (ROC) curves analyses were used to assess the diagnostic value of PBK in HCC (Fig. 3E-H). The result shows that PBK mRNA had high diagnostic potential to HCC from normal tissues 
in GSE76427(AUC=0.8799), GSE121248(AUC=0.9224), GSE62232(AUC=0.9975) and GSE84402 (AUC=0.9541).

\section{Relationships between PBK and clinicopathological characteristics of HCC patients}

The association between PBK mRNA expression and the clinicopathological characteristics of HCC patients including gender, race, tumor stage and age was investigated using TCGA data. Based on the median values of PBK mRNA level, 155 patients were classified as high PBK expression group, and 156 patients were classified as low PBK expression group. As shown in Fig.4, PBK expression was significantly correlated with race $(P=0.0024)$, tumor stage $(P=0.0089)$ and age $(P=0.0131)$, except for gender $(P=0.2998)$ in HCC patients.

\section{Survival analysis}

GEPIA and Kaplan-Meier Plotter were used for online survival data analysis. The results from GEPIA showed that the survival time of the group with high PBK expression was significantly shorter than that of the group with low PBK expression, both in OS (Logrank $P=0.0061$ ) and DFS (Logrank $P=0.006$ ) (Fig. $5 A \& B)$. According to the Kaplan-Meier Plotter website analysis for survival, patients having high PBK expressions were associated with poor prognosis compared with those having low PBK expression (OS: Logrank $P=4.8 \mathrm{e}-05$; DSS: Logrank $P=0.00086$; RFS: Logrank $P=0.00028$; PFS: Logrank $P=0.00066$ ) (Fig.5D-G).

The Kaplan-Meier analysis was performed to investigate the relationship between PBK expression and overall survival in $311 \mathrm{HCC}$ patients in TCGA cohort. Median expression of PBK of all samples was chosen as a cut-off to divide samples into the PBK-high $(n=155)$ group and PBK-low $(n=156)$ group. As shown in Fig. 5C, HCC patients with high PBK expression exhibited a poorer overall survival rate compared with the low-expression group $(P=0.007)$.

\section{Validation of PBK as an independent prognostic factor}

Using the Univariate Cox regression analysis, Tumor Stage $(\mathrm{HR}=2.389,95 \% \mathrm{Cl}=1.621-3.522, P<0.001)$ and PBK expression $(\mathrm{HR}=1.699,95 \% \mathrm{Cl}=1.154-2.502, P=0.007)$ were identified to be related to the overall survival of $311 \mathrm{HCC}$ patients in the TCGA training set. In the Multivariate Cox regression model, Tumor Stage $(\mathrm{HR}=2.274,95 \% \mathrm{Cl}=1.538-3.362, \mathrm{P}<0.001)$ and $\mathrm{PBK}$ expression $(\mathrm{HR}=1.566,95 \% \mathrm{Cl}=$ 1.062-2.311, $P=0.024$ ) were likewise confirmed to be independent prognostic factors of overall survival in HCC patients (Table 1). Overall, our results suggested that high PBK expression was an adverse prognostic factor and independent prognostic marker. 
Table 1. Univariable and multivariable Cox regression analyses of clinical characteristics.

\begin{tabular}{lllll}
\hline \multirow{2}{*}{ Variable } & \multicolumn{2}{l}{ Univariable Cox regression } & \multicolumn{2}{l}{ Multivariable Cox regression } \\
\cline { 2 - 5 } & $\mathrm{HR}(95 \% \mathrm{Cl})$ & $P$ value & $\mathrm{HR}(95 \% \mathrm{Cl})$ & $P$ value \\
\hline Gender & $0.872(0.717-1.061)$ & 0.173 & & \\
\hline Age & $0.811(0.552-1.191)$ & 0.285 & & \\
\hline Stage & $2.389(1.621-3.522)$ & $<0.001$ & $2.274(1.538-3.362)$ & $<0.001$ \\
\hline Race & $0.770(0.519-1.142)$ & 0.194 & & \\
\hline PBK & $1.699(1.154-2.502)$ & 0.007 & $1.566(1.062-2.311)$ & 0.024 \\
Co-expression and Regulatory Networks in HCC & &
\end{tabular}

We used GeneMANIA to construct a protein-protein interaction (PPI) network to analyze the interaction between PBK and other genes. PPI network showed that PBK significantly interacted with LRRC47, ARAF, LGALS9B, TTK, MELK and other essential genes. Biological function of these genes may include mitosis, organelle fission, G2/M transition of mitotic cell cycle, cell cycle G2/M phase transition, microtubule cytoskeleton organization, regulation of mitosis and nuclear division (Fig. 6).

In addition, the co-expression genes in TCGA-LIHC that are closely related to PBK were explored on the Linkedomics website. As shown in Fig.7A, 6869 positively correlated genes represented by red dots are closely related to PBK, while 3350 negatively correlated genes represented by green dots are closely related to PBK. The top 50 significant genes that positively and negatively correlated with PBK are shown in the heat map (Fig.7B\&C).

\section{Enrichment analysis of PBK functional networks in HCC}

GO and KEGG analysis of PBK and co-expression genes were further analyzed using the GSEA in Linkedomics (Fig.8). Consequently, the GO enrichment analysis revealed 1) In biological processes (BP), chromosome segregation, DNA replication, spindle organization, mitotic cell cycle phase transition, cell cycle checkpoint, protein activation cascade, peroxisomal transport, acute inflammatory response, peroxisome organization and antibiotic metabolic process were the most significant. 2) In cell composition (CC), condensed chromosome, chromosomal region, spindle, replication fork, midbody, blood microparticle, protein-lipid complex, respiratory chain, NADH dehydrogenase complex and mitochondrial protein complex were the most significant. 3) In molecular function (MF), catalytic activity acting on DNA, single-stranded DNA binding, damaged DNA binding, motor activity, helicase activity, oxidoreductase activity acting on $\mathrm{CH}-\mathrm{OH}$ group of donors, monooxygenase activity, tetrapyrrole binding, oxidoreductase activity acting on the $\mathrm{CH}-\mathrm{CH}$ group of donors and oxidoreductase activity acting on the aldehyde or oxo group of donors were the most significant. In the results of KEGG pathway analysis, PBK and co-expression genes were significantly enriched in cell cycle, DNA replication, oocyte meiosis, homologous recombination, fanconi anemia pathway, fatty acid degradation, valine, leucine and 
isoleucine degradation, drug metabolism, tyrosine metabolism and metabolism of xenobiotics by cytochrome P450.

\section{Correlation between tumor immune cell infiltration and PBK expression in HCC}

We evaluated the correlation between PBK expression in pan-caners and tumor-infiltrating lymphocytes (TILs), chemokines and receptors in the TISIDB database (Fig.9A, Fig.10A, Fig.11A). As shown in Fig.9B, PBK expression was positively closely related with infiltrating levels of Memory B cell (Mem_B) (rho=0.178, $\mathrm{P}=0.000577$ ), Type 2T helper cell (Th2) (rho=0.364, $\mathrm{P}=5.17 \mathrm{e}-13$ ), Effector memeory CD4 T cell (Tem_CD4) (rho=0.135, P=0.00885) and Activated CD4 T cell (Act_CD4) (rho=0.582 $\mathrm{P}<2.2 \mathrm{e}-16)$ in HCC. Negative correlation TILs include Monocyte, Effector memeory CD8 T cell (Tem_CD8), Type 1T helper cell (Th1) and so on. To receptors, PBK expression was positively closely related with CCR6 (rho $=0.123$, $P=0.0175)$ and CCR10 (rho $=0.175, P=0.000712)$, and was negatively correlated with CCR7 (rho $=-0.134$, $\mathrm{P}=0.0098$ ) and $\mathrm{CXCR} 1$ (rho $=-0.188, \mathrm{P}=0.000259$ ) (Fig.10B). In addition, the result showed that 12 types of chemokines were negatively correlated with the expression of PBK, and 6 types of chemokines were positive (Fig.11B).

In TIMER database, we investigated whether PBK was correlated with six main infiltrating immune cells (CD4+T cells, CD8+T cells, B cells, macrophages, neutrophils, and dendritic cells) in HCC. The results show that a significant positive correlation between PBK and CD4+T cells (Rho=0.149, $p=5.58 \mathrm{e}-03)$, CD8+T cells ( $R h o=0.145, p=6.97 e-03), B$ cells ( $R h o=0.386, p=1.13 e-13)$, macrophages (Rho=0.307, $p=6.05 e-09$ ), neutrophils (Rho $=0.172, p=1.32 e-03)$, and dendritic cells (Rho=0.477, $p=5.16 e-21)$ (Fig.12A). Moreover, we evaluated the relationship between PBK and six kinds of immune cells and the prognosis of HCC patients. We found that patients with high expression of PBK had a poor prognosis $(P=0.00181)$, which also confirmed our previous results. Furthermore, the expression of macrophages and neutrophils was positively correlated with poor prognosis (macrophages, $p=0.00837$; neutrophils, $p=0.00271$ ), meanwhile, there was no correlation between the remaining immune cells (CD4+T cells, CD8+T cells, $B$ cells and dendritic cells) and prognosis ( $P>0.05$ ) (Fig.12B). Multivariate Cox regression analysis showed that macrophages $(H R=10.217$, $P$ value $=0)$ and neutrophils $(H R=4.757, P$ value $=0.018)$ were also significant independent risk factors among the six types of immune cells (Table 2). 


\begin{tabular}{|llllll|}
\hline \multicolumn{7}{|l|}{ Table 2: Multivariate Cox analysis of immune cells infiltration } & & \\
\hline Cell type & Coef & HR & $95 \% \mathrm{Cl}$ & $P$ value & Sig \\
\hline T cell CD4+ & -0.667 & 0.513 & $(0.086-3.071)$ & 0.465 & \\
\hline T cell CD8+ & -0.036 & 0.965 & $(0.426-2.187)$ & 0.931 & \\
\hline B cell & -0.893 & 0.41 & $(0.055-3.066)$ & 0.385 & \\
\hline Macrophage & 2.324 & 10.217 & $(2.985-34.968)$ & 0.000 & $* \star *$ \\
\hline Neutrophil & 1.56 & 4.757 & $(1.303-17.361)$ & 0.018 & $*$ \\
\hline Dendritic cell & 0.557 & 1.745 & $(0.96-3.171)$ & 0.068 & \\
\hline Coef: regression coefficient; HR: hazard ratio; Cl: confidence interval; * $P<0.05, * \star \star P<0.001$ \\
\hline
\end{tabular}

\section{Discussion}

Although many people, especially those with chronic liver diseases, have realized the importance of early diagnosis of liver cancer, in the countries with high incidence rate of liver cancer, such as China,

Mongolia, Egypt, etc., most patients are diagnosed at late stages with poor prognosis. Surgery is the most successful treatment for HCC, either liver transplantation or liver resection depending on liver function, the presence of portal hypertension and tumor burden[27]. Unfortunately, postoperative recurrence rate remains high and there is no reliable prediction tool[28]. Liver transplantation is an ideal treatment for selected patients with HCC, as it removes both the tumor and potential cirrhosis, with 5-year survival rate exceeding $70 \%[29]$. However, due to tumor characteristics, patient co-morbidities or shortage of organs available for transplant, only $20 \%$ of patients can undergo curative treatment[30]. Locoregional therapies play an important role at all stages of HCC, aiming to increase overall survival while preserving liver function[31]. Percutaneous ablation has been shown to be a safe and effective treatment for early stage of HCC. Ablative techniques of various types are available, including percutaneous ethanol injection (PEI), radiofrequency ablation (RFA), microwave ablation (MWA), cryoablation (CRA), high intensity focused ultrasound (HIFU), laser ablation (LSA) and irreversible electroporation (IRE)[32]. Transcatheter arterial chemoembolization (TACE) is an effective method for the treatment of patients with intermediate-stage HCC, and has become the first choice for non-surgical treatment[33]. In fact, surgical or locoregional therapies can not meet the clinical needs of patients with HCC. Fortunately, sorafenib, the first targeted treatment drug, was launched in 2007, which opened the prelude of systematic treatment of HCC[34]. Systematic treatments for HCC changed in recent years with the advent of new targeted therapeutic agents and immunotherapy[35]. While these agents offer new hope for HCC patients, the optimal choice and sequence of treatment remain unknown, and without established biomarkers[36]. Therefore, it is very important to identify novel prognostic biomarkers and immuneassociated therapeutic targets in HCC patients. 
T-lymphokine-activated killer-cell-originated protein kinase (TOPK), also known as PDZ-binding kinase (PBK), is a novel mitotic serine/threonine protein kinase, which was confirmed to be associated with the development, progression, and metastasis of malignancies and to be a potential therapeutic target in cancer therapy[37]. Studies have shown that PBK expression is increased in various cancers compared with in normal tissues[38]. In this study, we explored the expression of PBK in pan-cancers using oncomine and TIMER databases. The result shown that PBK expression was extremely higher in most common tumor tissues, such as Colon adenocarcinoma, Cholangiocarcinoma, Breast invasive carcinoma, Liver hepatocellular carcinoma and so on. These conclusions suggest that the possibility of BPK to be a cancer therapeutic target. Then, mRNA expression profiles and clinicopathological data of HCC were downloaded from publicly available data repositories, including TCGA and GEO. Analysis results from HCCDB, GEPIA and HPA database showed that PBK increased significantly in HCC, both at the mRNA and protein levels. We also confirmed this with 4 independent GEO datasets- GSE84402, GSE76427, GSE62232 and GSE121248. To evaluate the prognostic value of PBK on the overall survival of HCC patients in TCGA cohort, the Kaplan-Meier and Cox regression analyses were performed. We found out that HCC patients with high PBK expression exhibited a poorer overall survival rate compared with the low-expression group. These results indicated that PBK could act as an independent prognostic marker in HCC. Previous studies showed that PBK, as a differentially expressed genes (DEGs), is highly expressed in $\mathrm{HCC}$ and is associated with poor prognosis, which is according with our results[39, 40]. However, our study attempted to deeper explore the relationships between PBK and clinicopathological characteristics of HCC patients. It's the first time to reveal that the expression of PBK is closely related to age, race and tumor stage. Patients were further stratified into subgroups of male and female, white or

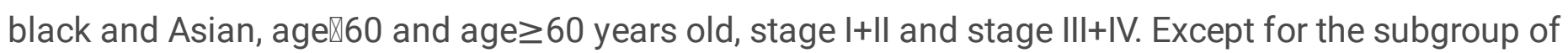
gender, the expression of PBK is higher in Asian patients under 60 with tumor stagellI+IV. The revelation of these results is helpful for the formulation of individualized treatment strategies with PBK as a therapeutic target. The receiver operator characteristic (ROC) curve and AUC estimates can determine for the expression of PBK to diagnose value of HCC. The ROC curves based on the TCGA database in AFPnegative HCC patients showed the AUC of PBK was 0.929[41]. In current study, the ROC curves analysis based on the GEO database shows that AUC of PBK in GSE84402, GSE76427, GSE62232 and GSE121248 was $0.9541,0.8799,0.9975$ and 0.9224 , respectively. Our results are in line with previous findings. ' In addition, the profiles of co-expression and regulator networks of PBK were analyzed. PPI network based on GeneMANIA showed that PBK significantly interacted with LRRC47, ARAF, LGALS9B, TTK, MELK and other essential genes. Biological function of these genes may include mitosis, organelle fission, G2/M transition of mitotic cell cycle, cell cycle G2/M phase transition, microtubule cytoskeleton organization, regulation of mitosis and nuclear division. We also obtained the co-expression genes in TCGA-LIHC that are closely related to PBK from Linkedomics. GSEA revealed the function of PBK and its related signal transduction pathway, such as cell cycle, DNA replication, amino acid metabolism and degradation, which might help explain the underlying molecular mechanisms of PBK in HCC. In the past few years, there have been few research reports that have the association between PBK with HCC. Functional analysis of PBK, derived in most cases from emerging high-throughput genomic and bioinformatics scanning approaches, is still unclear. The researchers identify PBK, a downstream effector 
of FoxM1, as an oncogene in HCC via the activation of $\beta$-Catenin signaling pathway in vitro and vivo[42]. Another study found PBK depletion suppressed migration and invasion of HCC cells and markedly inhibited the lung metastasis of HCC cells in orthotopic mouse model. Mechanistically, PBK enhanced uPAR expression by enhancing the binding of ETV4 to UPAR promoter to activate its transcription[4]. Cao et al, reported for the first time that overexpression of PBK relieved the apoptosis induced by Oxaliplatin and promoted the migration and invasion of Oxaliplatin-sensitive HCC cells[43].

Many studies about the possible role of PBK in tumor immune cell infiltration have emerged in recent years. In Esophageal squamous cell carcinoma, immune infiltration analysis showed that infiltration of dendritic cells was significantly negatively correlated with PBK expression levels[44]. Zhou et al. investigated the immune microenvironment in Ewing's sarcoma patients to identify immune-related gene signatures. PBK, one of 10 hub immune-related genes signature, was determined to exhibit independent prognostic significance for Ewing's sarcoma[45]. In HCC, the expression of PBK was significantly positively correlated with immune infiltration cells, including regulatory $T$ cells (Treg), $T$ follicular helper (TFH) cells, macrophages $\mathrm{MO}$, but negatively correlated with immune infiltration cells including monocytes[46]. In our study, the results from TIMER database shown that a significant positive correlation between PBK and six main infiltrating immune cells (CD4+T cells, CD8+T cells, B cells, macrophages, neutrophils, and dendritic cells). Multivariate Cox regression analysis showed that macrophages and neutrophils were also significant independent risk factors. We also found that PBK expression was positively closely related with infiltrating levels of Memory B cell (Mem_B), Type 2T helper cell (Th2), Effector memeory CD4 T cell (Tem_CD4) and Activated CD4 T cell (Act_CD4) in HCC, and was negatively correlated with Monocyte, Effector memeory CD8 T cell (Tem_CD8), Type 1T helper cell (Th1) and so on. Moreover, to our knowledge, it's the first time to reveal that the expression of PBK is closely related to chemokines and receptors. These findings are significant for further research about the detailed mechanisms of PBK in HCC. Multiple studies have shown that CCL20 is expressed in tumor cells and tumor-associated macrophages, which can activate CCR6 on cancer cells in an autocrine manner, causing their migration and epithelial-to-mesenchymal transition[47]. Interestingly, PBK expression was positively closely related with CCR6 and its ligand-CCL20 in HCC from our results, which suggest that PBK may be involved in the CCL20 $\rightarrow$ CCR6 axis, but still require further experimental validation. Similarly, our analysis shown that CCL19 and CCL21 combined with their receptor CCR7 were all negatively correlated with the expression of PBK. Meanwhile, researchers found that the increased expression of CCL19 and CCL21 in tumors can result infiltration of TILs and an improved prognosis for many tumor patients. This is consistent with our results. Highly expressed PBK may be negatively correlated with the CCL19/CCL21 $\rightarrow$ CCR7 axis and cause poor prognosis of liver cancer[47].

In conclusion, multiple studies have identified PBK as a crucial gene for HCC. The difference between our study and previous studies was that we reported the relationship between PBK expression and clinicopathological characteristics. We also identified the diagnostic value of PBK and as an independent prognostic factor in HCC. In addition, another advantage in our study was the first time to evaluate the correlation between PBK expression in pan-caners and tumor-infiltrating lymphocytes (TILs), chemokines and receptors. However, there were few limitations in the present study. Experimental verification is also 
needed to elucidate the molecular mechanisms in vivo and in vitro. Thus, results in our research may provide useful information for prospective research on HCC immunotherapy and targeted therapy.

\section{Abbreviations}

HCC: Hepatocellular carcinoma, PBK: PDZ-binding kinase, TCGA: The Cancer Genome Atlas, GEO: Gene Expression Omnibus, GEPIA: The Gene Expression Profiling Interactive Analysis, TIMER: Tumor Immune Estimation Resource, TISIDB: Tumor-Immune System Interaction Database, HPA: The human protein atlas database, ROC: Receiver operating characteristic, AUC: Area Under the Curve OS: Overall Survival, RFS: Recurrence-Free Survival, PFS: Progression-Free Survival, DSS: Disease-Specific Survival, GO: Gene Ontology, KEGG: Kyoto Encyclopedia of Genes and Genomes, BP: Biological processes, CC: Cellular components, MF: Molecular functions, GSEA: Gene Set Enrichment Analysis, TIICs: Tumor Infiltrating Immune Cells, TILs: tumor infiltrating lymphocytes.

\section{Declarations}

\section{Acknowledgements}

The authors would like to sincerely thank Department of neurosurgery, The first affiliated hospital of Shanxi medical university and Department of interventional radiology, Shanxi province people's hospital for valuable help in our study.

\section{Funding}

This work was supported by Scientific Research Project of Shanxi Provincial Health and Family Planning Commission (2015022); Key R\&D Projects of Shanxi Province (201803031149).

\section{Authors' contributions}

Wei Mu and Jingxian Zhang is responsible for writing and submitting the papers; Yaoli Xie and Runzhi Yan is responsible for data analysis and collation; Jinhu Li is responsible for the production of pictures; Yimin Fan and Yu'e Liu is responsible for the manuscript fees and ideas guidance. The authors have read and approved the final manuscript.

\section{Availability of data and materials}

The data used to support the findings of this study are included within the article.

\section{Ethics approval and consent to participate}

There were no cell, tissue, or animal studies. No ethical requirements are involved.

\section{Consent for publication}


All authors agree to publish the paper. All clinical records in this study were obtained with the approval of Institutional Review Boards and the need for patient consent was waived following Institutional Review Board protocol review.

Competing interests

The authors declare that they have no competing interests.

\section{References}

1. Singal AG, Lampertico P, Nahon P: Epidemiology and surveillance for hepatocellular carcinoma: New trends. J Hepatol 2020, 72:250-261.

2. Bray F, Ferlay J, Soerjomataram I, Siegel RL, Torre LA, Jemal A: Global cancer statistics 2018: GLOBOCAN estimates of incidence and mortality worldwide for 36 cancers in 185 countries. CA Cancer $J$ Clin 2018, 68:394-424.

3. Rimassa L, Pressiani T, Merle P: Systemic Treatment Options in Hepatocellular Carcinoma. Liver Cancer 2019, 8:427-446.

4. Yang QX, Zhong S, He L, Jia XJ, Tang H, Cheng ST, Ren JH, Yu HB, Zhou L, Zhou HZ, et al: PBK overexpression promotes metastasis of hepatocellular carcinoma via activating ETV4-uPAR signaling pathway. Cancer Lett 2019, 452:90-102.

5. Hu F, Gartenhaus RB, Eichberg D, Liu Z, Fang HB, Rapoport AP: PBK/TOPK interacts with the DBD domain of tumor suppressor p53 and modulates expression of transcriptional targets including p21. Oncogene 2010, 29:5464-5474.

6. Dong C, Fan W, Fang S: PBK as a Potential Biomarker Associated with Prognosis of Glioblastoma. 2020, 70:56-64.

7. Dou X, Wei J, Sun A, Shao G, Childress C, Yang W, Lin Q: PBK/TOPK mediates geranylgeranylation signaling for breast cancer cell proliferation. Cancer Cell Int 2015, 15:27.

8. Shih MC, Chen JY, Wu YC, Jan YH, Yang BM, Lu PJ, Cheng HC, Huang MS, Yang CJ, Hsiao M, Lai JM: TOPK/PBK promotes cell migration via modulation of the PI3K/PTEN/AKT pathway and is associated with poor prognosis in lung cancer. Oncogene 2012, 31:2389-2400.

9. Lei B, Liu S, Qi W, Zhao Y, Li Y, Lin N, Xu X, Zhi C, Mei J, Yan Z, et al: PBK/TOPK expression in nonsmall-cell lung cancer: its correlation and prognostic significance with Ki67 and p53 expression. Histopathology 2013, 63:696-703.

10. Ma H, Li Y, Wang X, Wu H, Qi G, Li R, Yang N, Gao M, Yan S, Yuan C, Kong B: PBK, targeted by EVl1, promotes metastasis and confers cisplatin resistance through inducing autophagy in high-grade serous 
ovarian carcinoma. Cell Death Dis 2019, 10:166.

11. Chang CF, Chen SL, Sung WW, Hsieh MJ, Hsu HT, Chen LH, Chen MK, Ko JL, Chen CJ, Chou MC: PBK/TOPK Expression Predicts Prognosis in Oral Cancer. Int J Mol Sci 2016, 17.

12. Warren AY, Massie CE: A reciprocal feedback between the PDZ binding kinase and androgen receptor drives prostate cancer. 2019, 38:1136-1150.

13. Ohashi T, Komatsu S, Ichikawa D, Miyamae M, Okajima W, Imamura T, Kiuchi J, Kosuga T, Konishi H, Shiozaki A, et al: Overexpression of PBK/TOPK relates to tumour malignant potential and poor outcome of gastric carcinoma. Br J Cancer 2017, 116:218-226.

14. Cerami E, Gao J, Dogrusoz U, Gross BE, Sumer SO, Aksoy BA, Jacobsen A, Byrne CJ, Heuer ML, Larsson $\mathrm{E}$, et al: The cBio cancer genomics portal: an open platform for exploring multidimensional cancer genomics data. Cancer Discov 2012, 2:401-404.

15. Gao J, Aksoy BA, Dogrusoz U, Dresdner G, Gross B, Sumer SO, Sun Y, Jacobsen A, Sinha R, Larsson $\mathrm{E}$, et al: Integrative analysis of complex cancer genomics and clinical profiles using the cBioPortal. Sci Signal 2013, 6:pl1.

16. Rhodes DR, Kalyana-Sundaram S, Mahavisno V, Varambally R, Yu J, Briggs BB, Barrette TR, Anstet MJ, Kincead-Beal C, Kulkarni P, et al: Oncomine 3.0: genes, pathways, and networks in a collection of 18,000 cancer gene expression profiles. Neoplasia 2007, 9:166-180.

17. Li T, Fan J, Wang B, Traugh N, Chen Q, Liu JS, Li B, Liu XS: TIMER: A Web Server for Comprehensive Analysis of Tumor-Infiltrating Immune Cells. Cancer Res 2017, 77:e108-e110.

18. Li B, Severson E, Pignon JC, Zhao H, Li T, Novak J, Jiang P, Shen H, Aster JC, Rodig S, et al: Comprehensive analyses of tumor immunity: implications for cancer immunotherapy. Genome Bio/ 2016, 17:174.

19. Lian Q, Wang S, Zhang G, Wang D, Luo G, Tang J, Chen L, Gu J: HCCDB: A Database of Hepatocellular Carcinoma Expression Atlas. Genomics Proteomics Bioinformatics 2018, 16:269-275.

20. Tang Z, Li C, Kang B, Gao G, Li C, Zhang Z: GEPIA: a web server for cancer and normal gene expression profiling and interactive analyses. Nucleic Acids Res 2017, 45:W98-w102.

21. Uhlén M, Fagerberg L, Hallström BM, Lindskog C, Oksvold P, Mardinoglu A, Sivertsson Å, Kampf C, Sjöstedt E, Asplund A, et al: Proteomics. Tissue-based map of the human proteome. Science 2015, 347:1260419.

22. Menyhárt $\mathrm{O}$, Nagy Á: Determining consistent prognostic biomarkers of overall survival and vascular invasion in hepatocellular carcinoma. 2018, 5:181006. 
23. Franz M, Rodriguez H, Lopes C, Zuberi K, Montojo J, Bader GD, Morris Q: GeneMANIA update 2018. Nucleic Acids Res 2018, 46:W60-w64.

24. Vasaikar SV, Straub P, Wang J, Zhang B: LinkedOmics: analyzing multi-omics data within and across 32 cancer types. Nucleic Acids Res 2018, 46:D956-d963.

25. Li T, Fu J, Zeng Z, Cohen D, Li J, Chen Q, Li B, Liu XS: TIMER2.0 for analysis of tumor-infiltrating immune cells. Nucleic Acids Res 2020, 48:W509-w514.

26. Ru B, Wong CN, Tong Y, Zhong JY, Zhong SSW, Wu WC, Chu KC, Wong CY, Lau CY, Chen I, et al: TISIDB: an integrated repository portal for tumor-immune system interactions. Bioinformatics 2019, 35:4200-4202.

27. Johnston MP, Khakoo SI: Immunotherapy for hepatocellular carcinoma: Current and future. World J Gastroenterol 2019, 25:2977-2989.

28. Ji GW, Zhu FP, Xu Q, Wang K, Wu MY, Tang WW, Li XC, Wang XH: Machine-learning analysis of contrastenhanced $\mathrm{CT}$ radiomics predicts recurrence of hepatocellular carcinoma after resection: A multiinstitutional study. EBioMedicine 2019, 50:156-165.

29. Rich NE, Parikh ND, Singal AG: Hepatocellular Carcinoma and Liver Transplantation: Changing Patterns and Practices. Curr Treat Options Gastroenterol 2017, 15:296-304.

30. Ghinolfi D, Rreka E, Pezzati D, Filipponi F, De Simone P: Perfusion machines and hepatocellular carcinoma: a good match between a marginal organ and an advanced disease? Trans/ Gastroentero/ Hepatol 2017, 2:87.

31. Cardarelli-Leite L, Hadjivassiliou A, Klass D, Chung J, Ho SGF, Lim HJ, Kim PTW, Mujoomdar A, Liu DM: Current locoregional therapies and treatment strategies in hepatocellular carcinoma. Curr Oncol 2020, 27:S144-S151.

32. Shiina S, Sato K, Tateishi R, Shimizu M, Ohama H, Hatanaka T, Takawa M, Nagamatsu H, Imai Y: Percutaneous Ablation for Hepatocellular Carcinoma: Comparison of Various Ablation Techniques and Surgery. Can J Gastroenterol Hepatol 2018, 2018:4756147.

33. Chen Z, Xie H, Hu M, Huang T, Hu Y, Sang N, Zhao Y: Recent progress in treatment of hepatocellular carcinoma. Am J Cancer Res 2020, 10:2993-3036.

34. Marisi G, Cucchetti A, Ulivi P, Canale M, Cabibbo G, Solaini L, Foschi FG, De Matteis S, Ercolani G, Valgiusti $\mathrm{M}$, et al: Ten years of sorafenib in hepatocellular carcinoma: Are there any predictive and/or prognostic markers? World J Gastroentero/ 2018, 24:4152-4163.

35. Wang T, Zhang Q, Wang N, Liu Z, Zhang B, Zhao Y: Research Progresses of Targeted Therapy and Immunotherapy for Hepatocellular Carcinoma. Curr Med Chem 2021, 28:3107-3146. 
36. Chen B, Garmire L, Calvisi DF, Chua MS, Kelley RK, Chen X: Harnessing big 'omics' data and Al for drug discovery in hepatocellular carcinoma. Nat Rev Gastroenterol Hepatol 2020, 17:238-251.

37. Han Z, Li L, Huang Y, Zhao H, Luo Y: PBK/TOPK: A Therapeutic Target Worthy of Attention. Cells 2021, 10.

38. Huang H, Lee MH, Liu K, Dong Z, Ryoo Z, Kim MO: PBK/TOPK: An Effective Drug Target with Diverse Therapeutic Potential. Cancers (Basel) 2021, 13.

39. Zhou Z, Li Y, Hao H, Wang Y, Zhou Z, Wang Z, Chu X: Screening Hub Genes as Prognostic Biomarkers of Hepatocellular Carcinoma by Bioinformatics Analysis. Cell Transplant 2019, 28:76S-86S.

40. Meng Z, Wu J, Liu X, Zhou W, Ni M, Liu S, Guo S, Jia S, Zhang J: Identification of potential hub genes associated with the pathogenesis and prognosis of hepatocellular carcinoma via integrated bioinformatics analysis. J Int Med Res 2020, 48:300060520910019.

41. Liu Z, Pu Y, Bao Y, He S: Investigation of Potential Molecular Biomarkers for Diagnosis and Prognosis of AFP-Negative HCC. Int J Gen Med 2021, 14:4369-4380.

42. Yang YF, Pan YH, Cao Y, Fu J, Yang X, Zhang MF, Tian QH: PDZ binding kinase, regulated by FoxM1, enhances malignant phenotype via activation of beta-Catenin signaling in hepatocellular carcinoma. Oncotarget 2017, 8:47195-47205.

43. Cao H, Yang M, Yang Y, Fang J, Cui Y: PBK/TOPK promotes chemoresistance to oxaliplatin in hepatocellular carcinoma cells by regulating PTEN. Acta Biochim Biophys Sin (Shanghai) 2021, 53:584592.

44. Zheng L, Li L, Xie J, Jin H, Zhu N: Six Novel Biomarkers for Diagnosis and Prognosis of Esophageal squamous cell carcinoma: validated by scRNA-seq and qPCR. J Cancer 2021, 12:899-911.

45. Zhou Y, Xu B, Wu S, Liu Y: Prognostic Immune-Related Genes of Patients With Ewing's Sarcoma. Front Genet 2021, 12:669549.

46. Huang R, Liu J, Li H, Zheng L, Jin H, Zhang Y, Ma W, Su J, Wang M, Yang K: Identification of Hub Genes and Their Correlation With Immune Infiltration Cells in Hepatocellular Carcinoma Based on GEO and TCGA Databases. Front Genet 2021, 12:647353.

47. Korbecki J, Grochans S, Gutowska I, Barczak K, Baranowska-Bosiacka I: CC Chemokines in a Tumor: A Review of Pro-Cancer and Anti-Cancer Properties of Receptors CCR5, CCR6, CCR7, CCR8, CCR9, and CCR10 Ligands. Int J Mol Sci 2020, 21.

\section{Figures}


A

Disease Summary for PBK

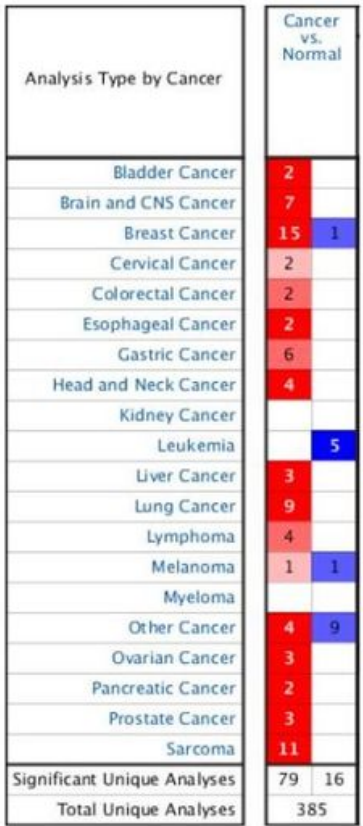

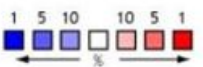

B

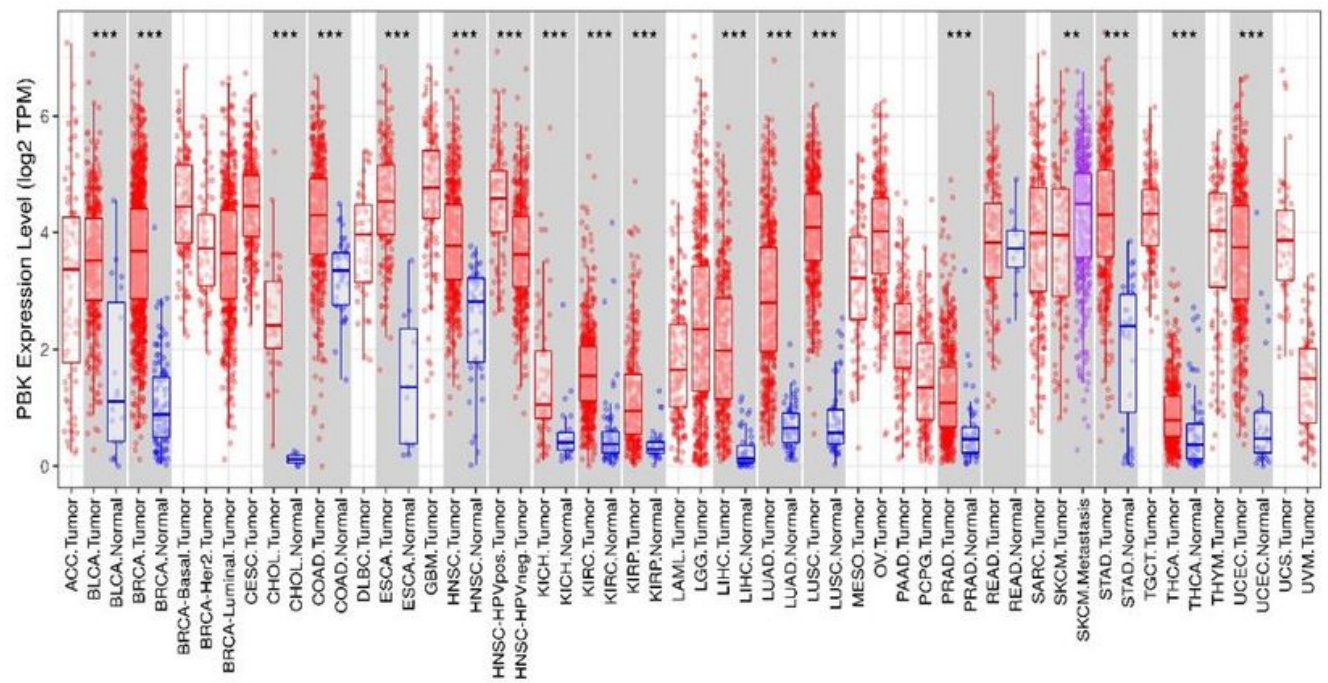

\section{Figure 1}

The expression of PBK in pan-cancers analysis within the Oncomine database and Timer. A: PBK mRNA levels in 20 types of human cancers analyzed by Oncomine. Red means increased expression and blue means decreased expression. The numbers indicated the amounts of dataset satisfying the threshold in the colored cell. B: PBK mRNA levels analyzed by TIMER. $\left({ }^{\star \star *} \mathrm{P}<0.001,{ }^{\star \star} \mathrm{P}<0.01,{ }^{*} \mathrm{P}<0.05\right)$ 
A

\begin{tabular}{|c|c|c|c|c|c|c|}
\hline Dotaset & P.ratur & Tppe & Nums. & Hean & STO & IQR \\
\hline \multirow{2}{*}{ necoen } & \multirow{2}{*}{$12300-5 s$. } & HOC & 100 & 790 & $1+177$ & 1570 \\
\hline & & Adpacent & nr & 4230 & 1056 & 1.006 \\
\hline \multirow{4}{*}{ Recoes } & \multirow{4}{*}{$27500-49$} & nos & 265 & 0.7054 & 0.0005 & 0.6063 \\
\hline & & Aqpoent & 243 & 0.05644 & conse & 0.65700 \\
\hline & & cintore & 40 & 01099 & 0 งเล & 000000 \\
\hline & & Meuthy & 6 & 0.00150 & $001 m^{2}$ & oeans \\
\hline \multirow{2}{*}{ Hecoes 4} & \multirow{2}{*}{2040054} & HOC & 240 & 5762 & 0.747 & 1072 \\
\hline & & Adpramit & 193 & 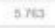 & $0 . \sin t$ & 0 ras \\
\hline \multirow{2}{*}{ necoes } & \multirow{2}{*}{$3740 e-60$} & HOC & 225 & $5+59$ & 1062 & 1681 \\
\hline & & Adjecromf & 200 & 2500 & 020000 & 02142 \\
\hline \multirow{2}{*}{ MCCDEN" } & \multirow{2}{*}{ orano } & HOC & 88 & 5.906 & 0.062 & 1,022 \\
\hline & & Adpacent & 40 & 5950 & 1015 & 1257 \\
\hline \multirow{2}{*}{ Mecou12 } & \multirow{2}{*}{$2,1500 \cdot 12$} & MCC & ar & 6.962 & 150 & 2030 \\
\hline & & Adipcent & 10 & 5915 & 12001 & 1810 \\
\hline \multirow[t]{2}{*}{ Hecosis } & \multirow[t]{2}{*}{ ortsoness } & noc & 220 & $48 \pi$ & 07895 & 0.7515 \\
\hline & & Appisent & 168 & 2967 & 02154 & 02037 \\
\hline \multirow{2}{*}{ HeCOQ16 } & \multirow{2}{*}{$1.3000-22$} & HOC & 309 & 5748 & 1.554 & 2020 \\
\hline & & Adpecent & as & 3028 & 1.625 & 22000 \\
\hline \multirow{2}{*}{ Hecosese } & \multirow{2}{*}{$59900-10$} & $H O C$ & 60 & $4366^{\circ}$ & 0.495 & $1+1+97$ \\
\hline & & Adspocent & 100 & $x+158$ & 02095 & 02017 \\
\hline \multirow{2}{*}{ Heedert? } & \multirow{2}{*}{$29300-20$} & MOC & ns & actos & 0 oseas & $1 \mathrm{no}$ \\
\hline & & Adpocamt & 02 & 7006 & 0.30113 & 030000 \\
\hline \multirow{2}{*}{ Heconis } & \multirow{2}{*}{$51900-64$} & HOC & 212 & 1844 & 09125 & 1245 \\
\hline & & Adpremt & 177 & 03510 & 03456 & 0.2500 \\
\hline
\end{tabular}

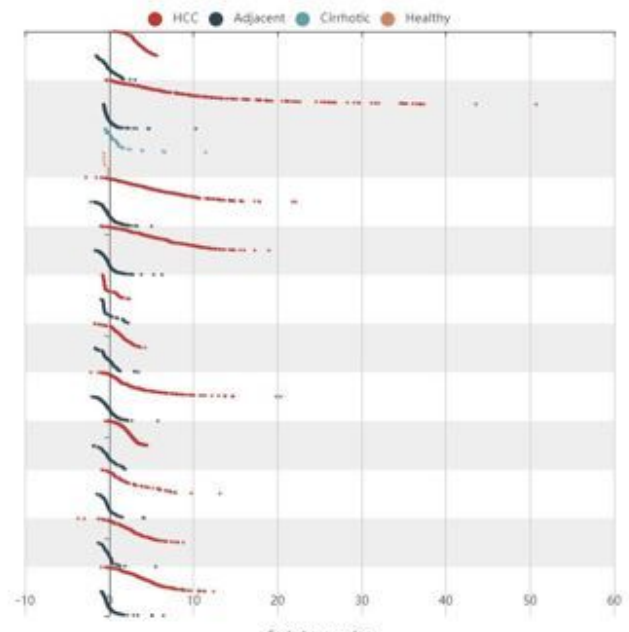

B

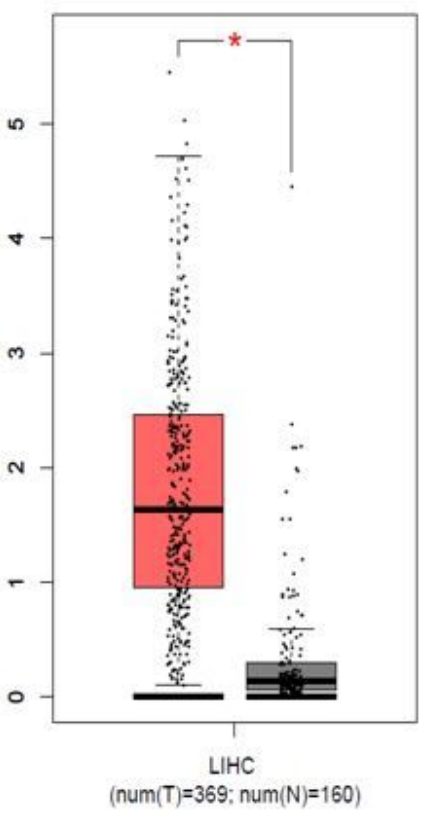

C

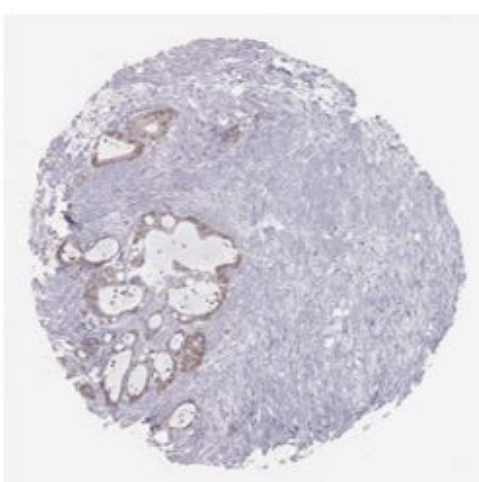

$\mathrm{HCC}$

Staining: Medium

Intensity: Moderate

Quantity: 75\%-25\%

Location:

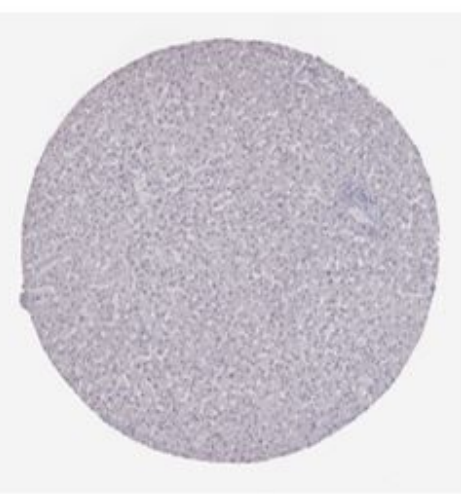

Hepatocytes

Staining: Not detected

Intensity: Negative

Quantity: None

Location: None

Cytoplasmic/membranous, nuclear

\section{Figure 2}

The expression level of PBK is upregulated in HCC. A: The expression of PBK in HCC tissues in 10 datasets from HCCDB. B: PBK expression profile in HCC based on GEPIA database. $\left({ }^{*} P<0.05\right)$ C: Protein expression and distribution of PBK from HPA. 
A

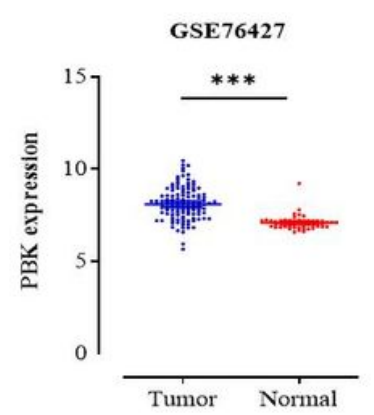

$\mathrm{E}$

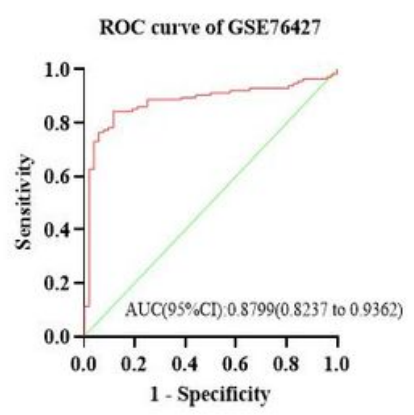

B

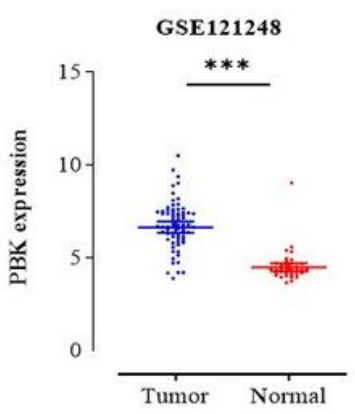

$\mathrm{F}$

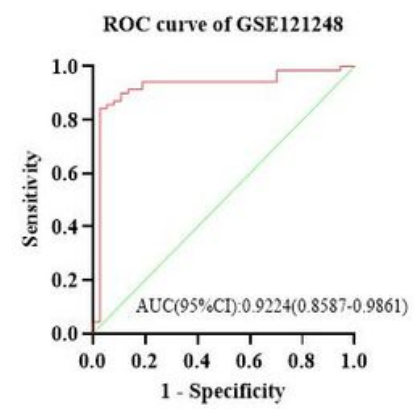

$\mathrm{C}$

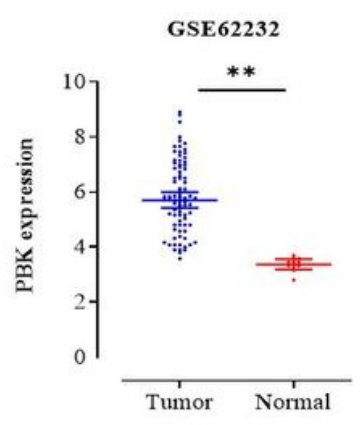

G

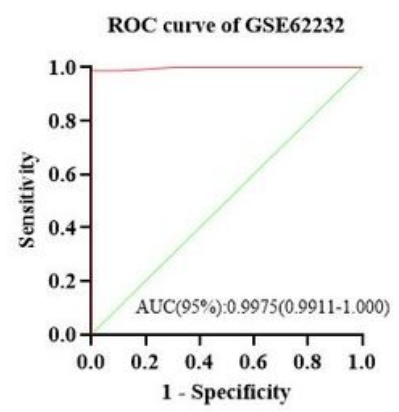

$\mathrm{D}$

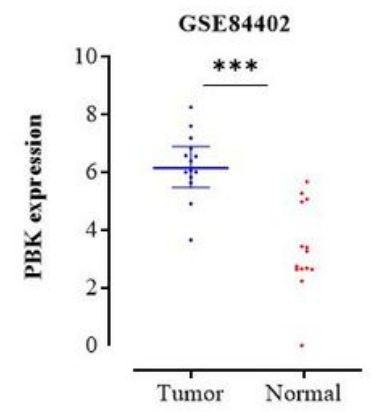

$\mathrm{H}$

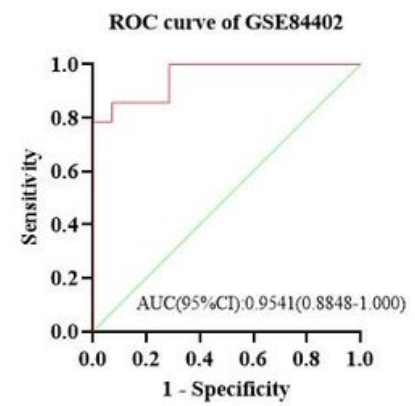

\section{Figure 3}

The expression and diagnostic significance of PBK in GEO database. Levels of PBK are statistically higher in HCC than that in normal tissues in GSE76427(A), GSE12148(B), GSE62232(C) and GSE84402(D). $\left({ }^{\star *} * P<0.001,{ }^{*} P<0.01, * P<0.05\right)$ ROC curves of $P B K$ for the identification of HCC were meaningful in GSE76427(E), GSE12148(F), GSE62232(G) and GSE84402(H) $(P<0.001)$.

A

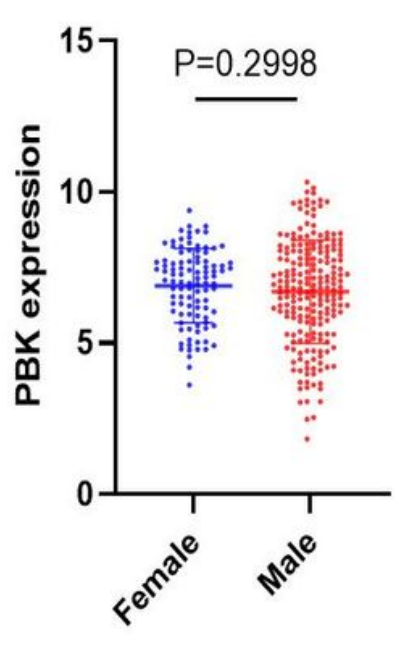

B

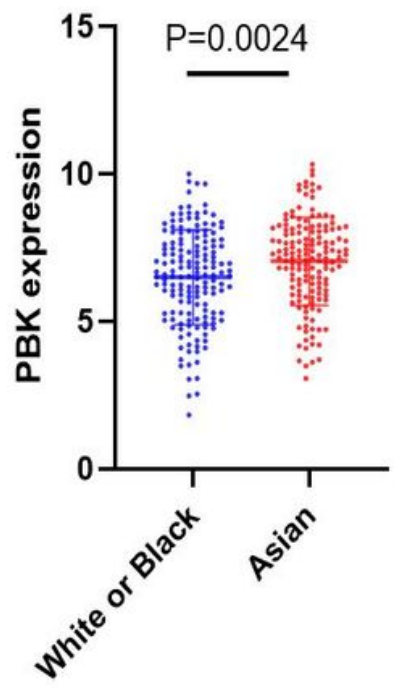

C

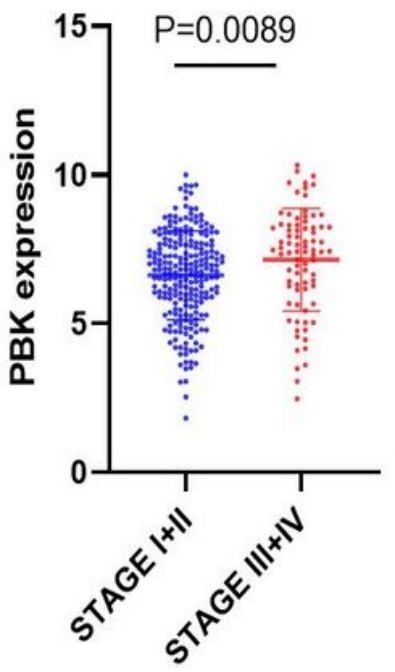

D

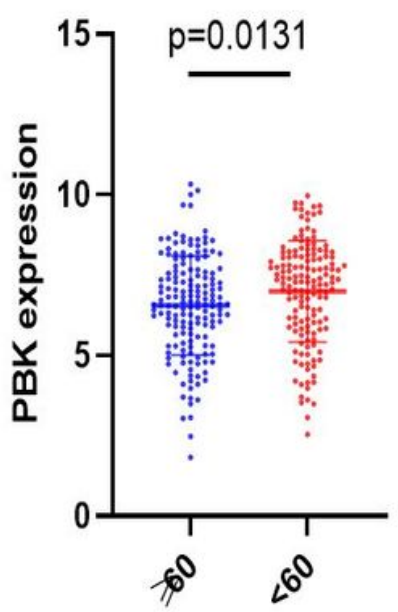

Figure 4 
Relationships between PBK mRNA levels and clinical pathological characteristics. PBK mRNA levels was significantly correlated with race(B), tumor stage(C) and age(D), except for gender(A).

A

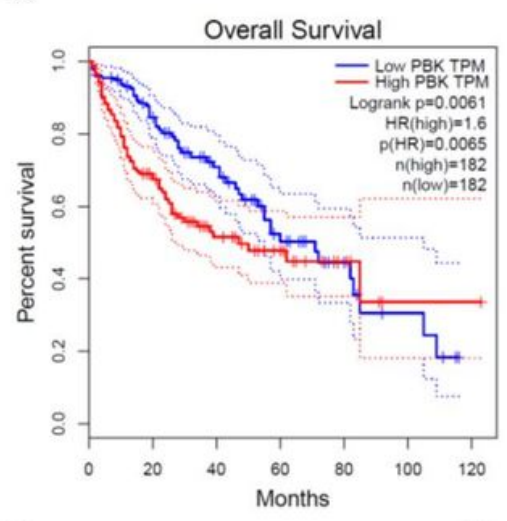

$\mathrm{D}$

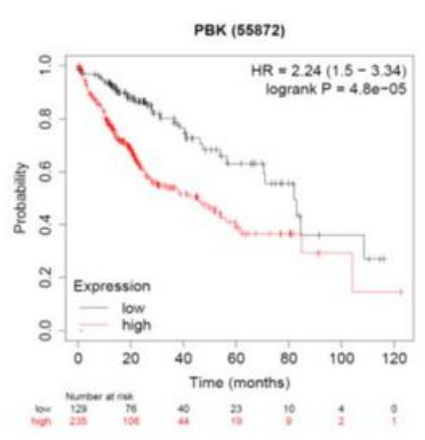

B

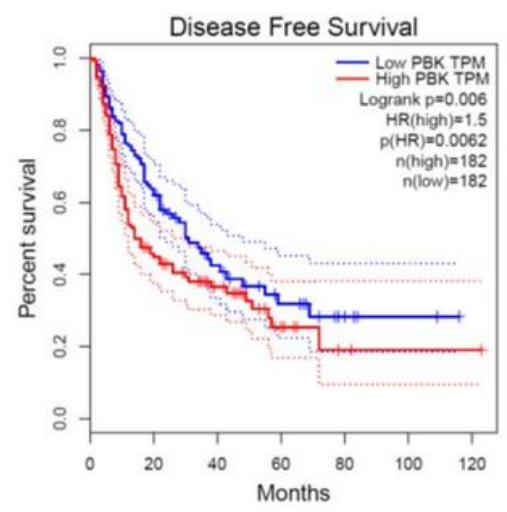

$\mathrm{F}$
$\mathrm{C}$

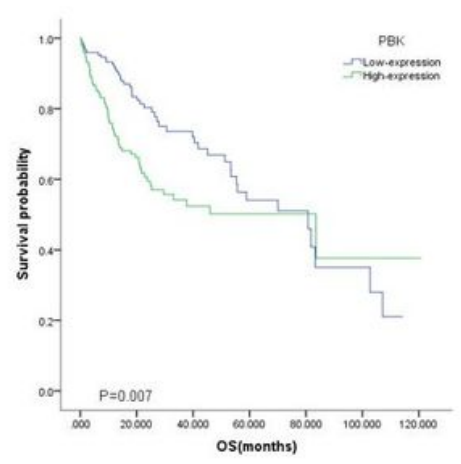

G
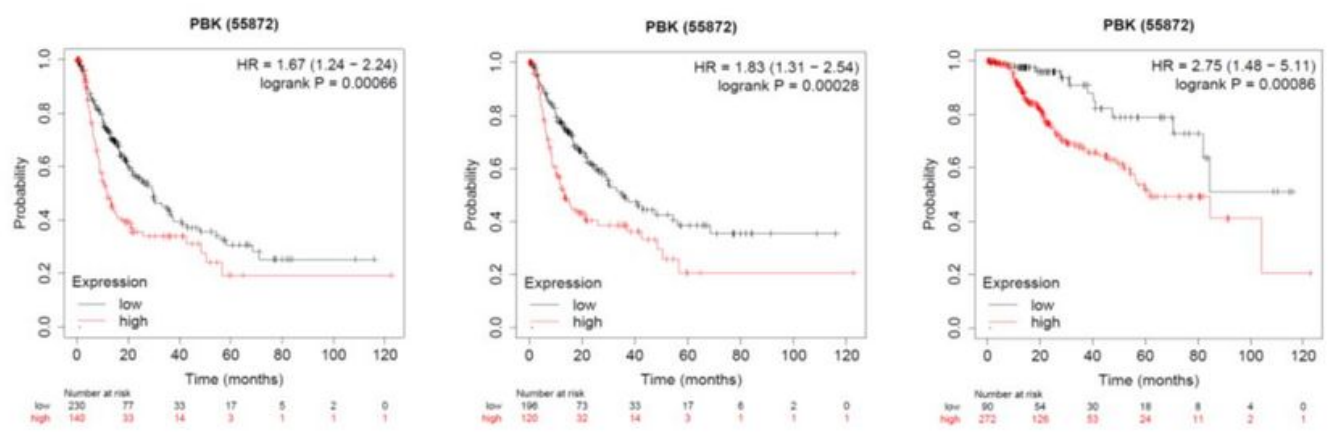

\section{Figure 5}

Relationship between PBK expression and poor prognosis in HCC. A: Effect of PBK expression on HCC Overall Survival from GEPIA; B: Effect of PBK expression on HCC Disease Free Survival from GEPIA; C: Kaplan-Meier survival curves indicated that high PBK mRNA expression had a shorter OS than those with low-level of PBK from TCGA-LIHC database; D-G: Effect of PBK expression on HCC survival from KaplanMeier Plotter (D: OS, E: DSS, F: RFS, G: PFS) 


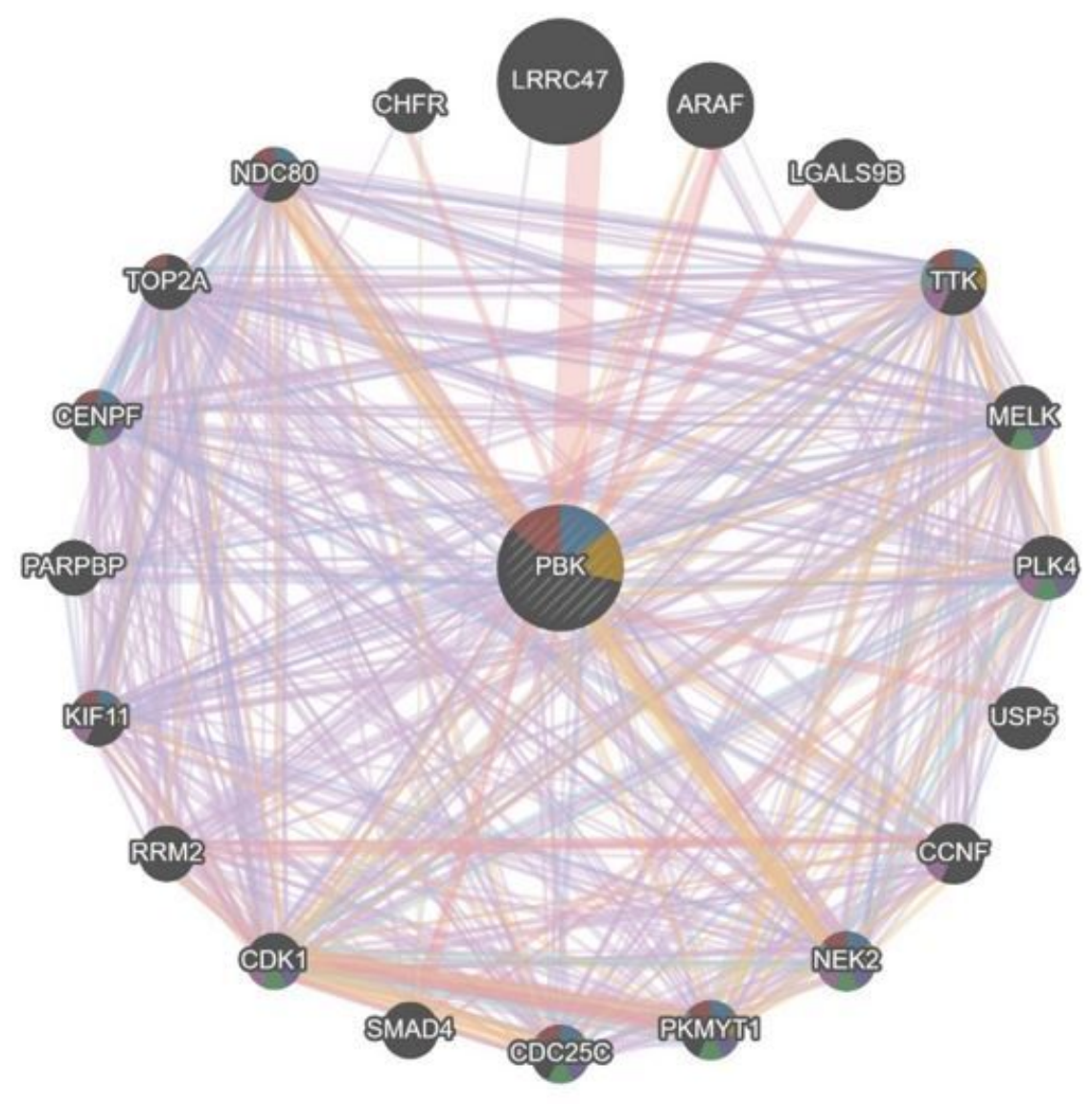

\section{Functions}

mitosis

organelle fission

G2/M transition of mitotic cell cycle

cell cycle G2/M phase transition

microtubule cytoskeleton organization regulation of mitosis

nuclear division

\section{Networks}

Physical Interactions

Co-expression

Predicted

Co-localization

Pathway

Genetic Interactions

Shared protein domains

\section{Figure 6}

Fig.6 Protein-protein interaction (PPI) network and functional analysis showing the gene set enriched in the target network of PBK (GeneMANIA). Distinct colors of the network edge indicate the bioinformatics methods applied: physical interactions, co-expression, predicted, co-localization, pathway, genetic interactions, and shared protein domains. The distinct colors for the network nodes show the biological functions of the sets of enrichment genes.

A

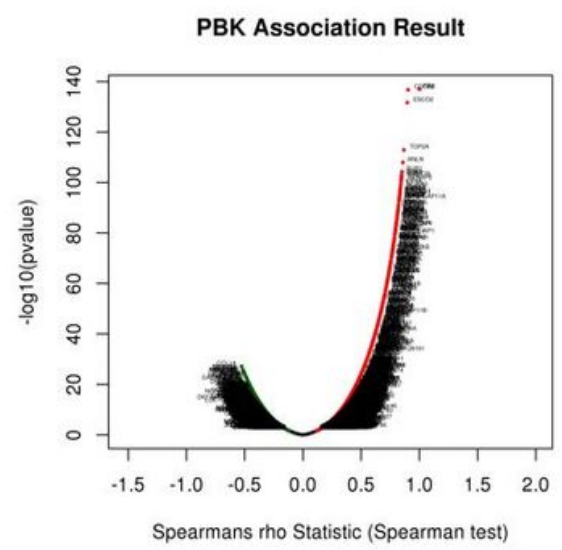

$\mathrm{B}$

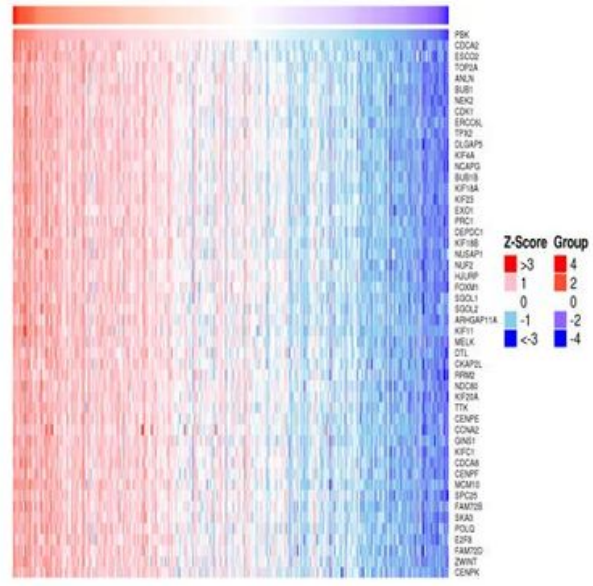

$\mathrm{C}$

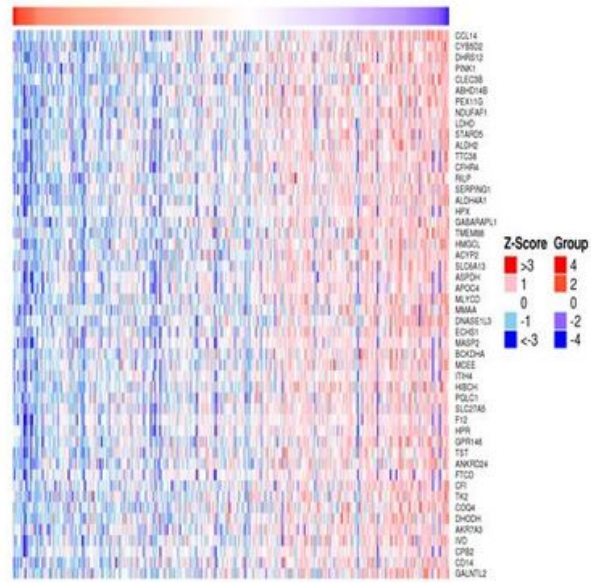

Figure 7 
Genes differentially expressed in correlation with PBK in HCC (LinkedOmics). (A) The volcanic map showed correlations between PBK and genes differentially expressed in HCC. Red indicates positively correlated genes, and green indicates negatively correlated genes. (B-C) Heat maps showing genes positively and negatively correlated with PBK in HCC (TOP 50).
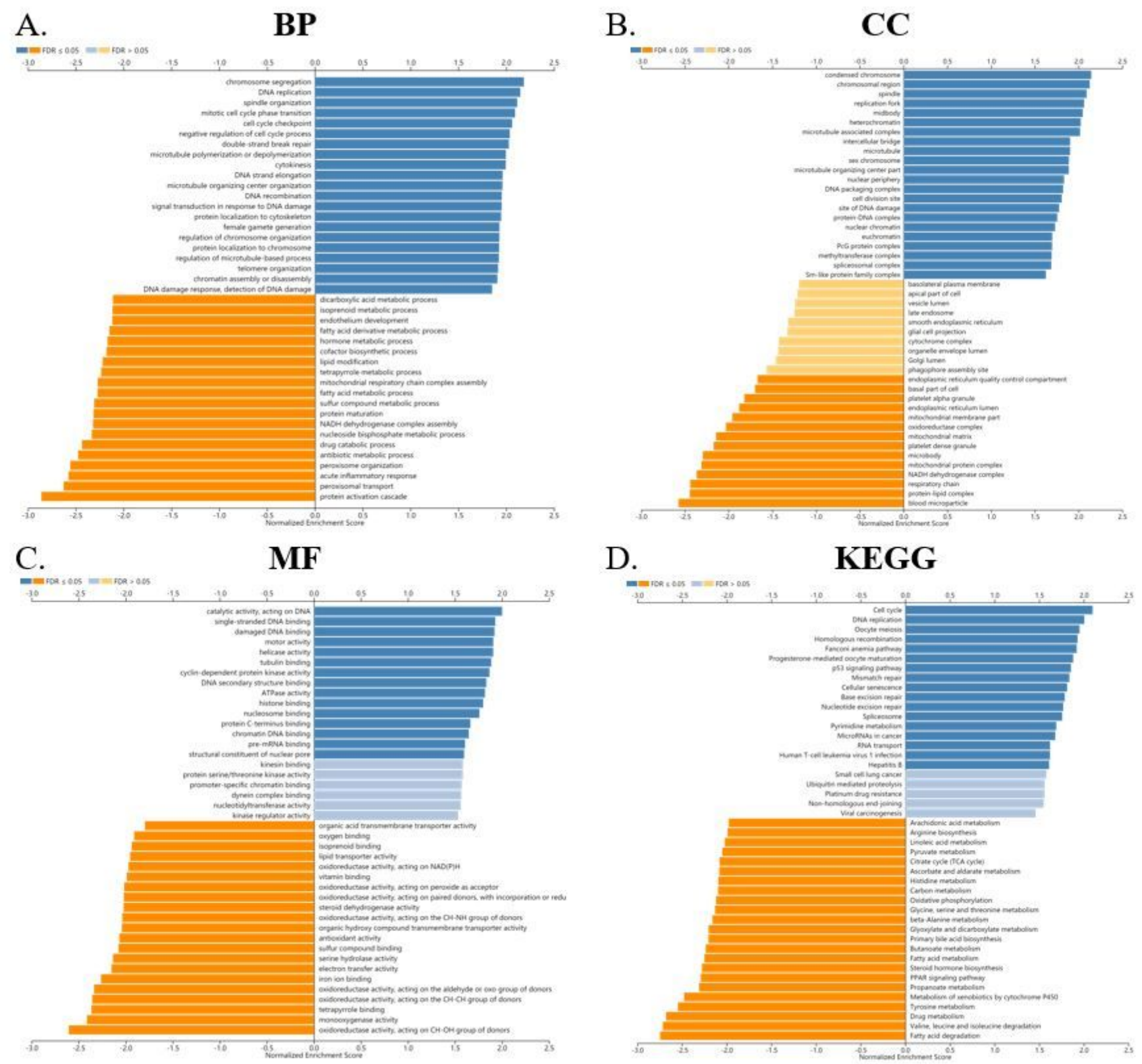

\section{Figure 8}

GO annotations and KEGG pathways of PBK in HCC (LinkedOmics). (A) Biological processes. (B) Cellular components. (C) Molecular functions. (D) KEGG pathway analysis. 
A

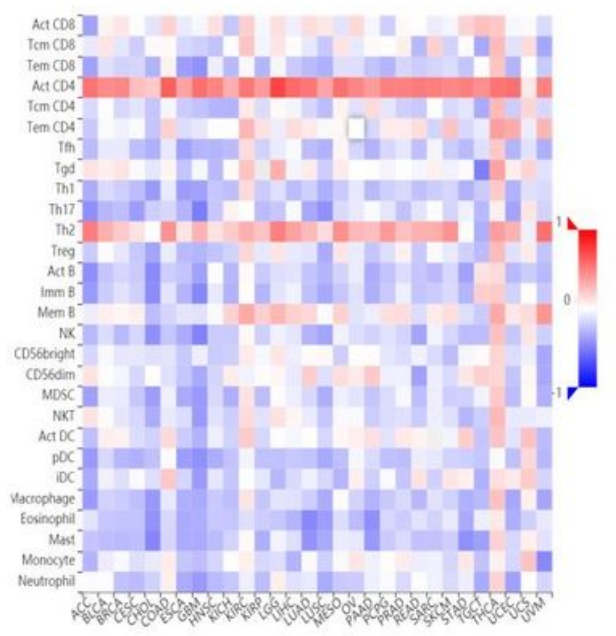

B
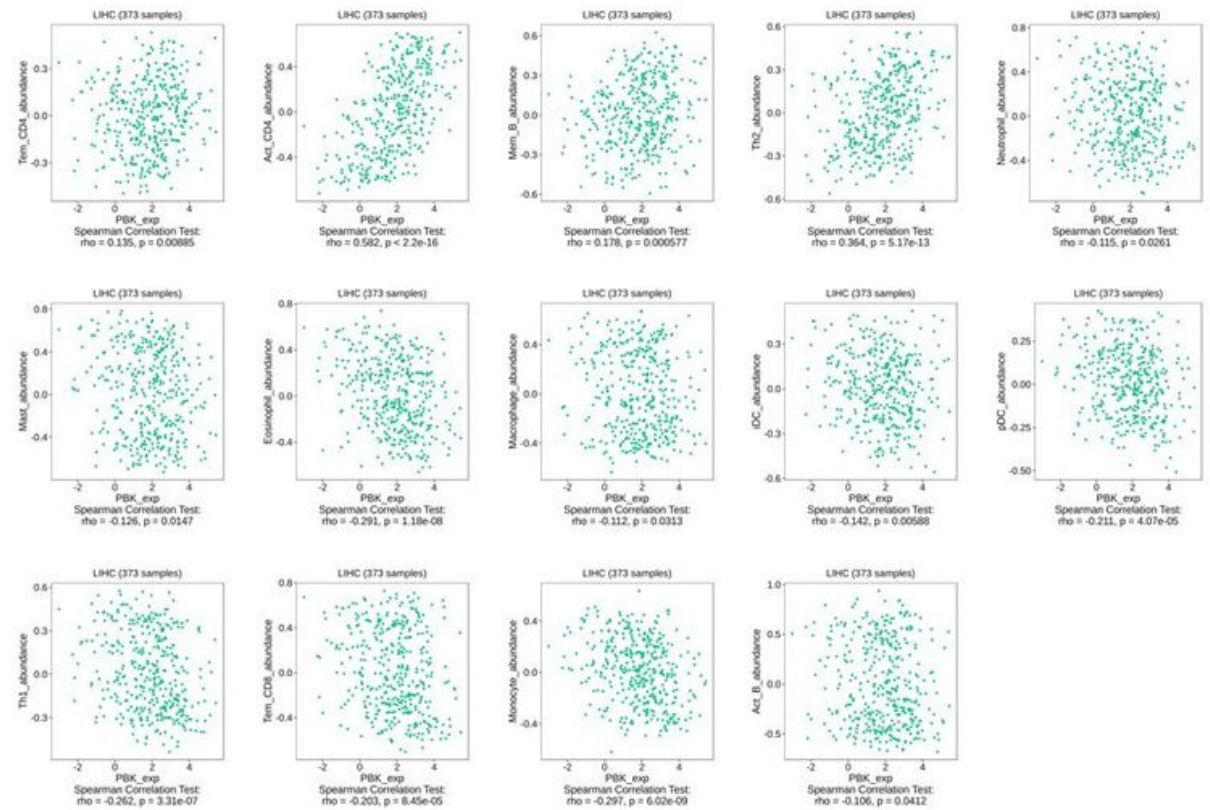

\section{Figure 9}

A: Relations between abundance of tumor-infiltrating lymphocytes (TILs) and expression of PBK in pancancers (red is positive correlated and blue is negative correlated). B: PBK expression was positively closely related with infiltrating levels of Memory B cell (Mem_B), Type 2T helper cell (Th2), Effector memeory CD4 T cell (Tem_CD4) and Activated CD4 T cell (Act_CD4) in HCC, and was negatively correlated with Monocyte, Effector memeory CD8 T cell (Tem_CD8), Type 1T helper cell (Th1) and so on. (rho $>0$ indicate positively, rho $<0$ indicate negatively) 
A

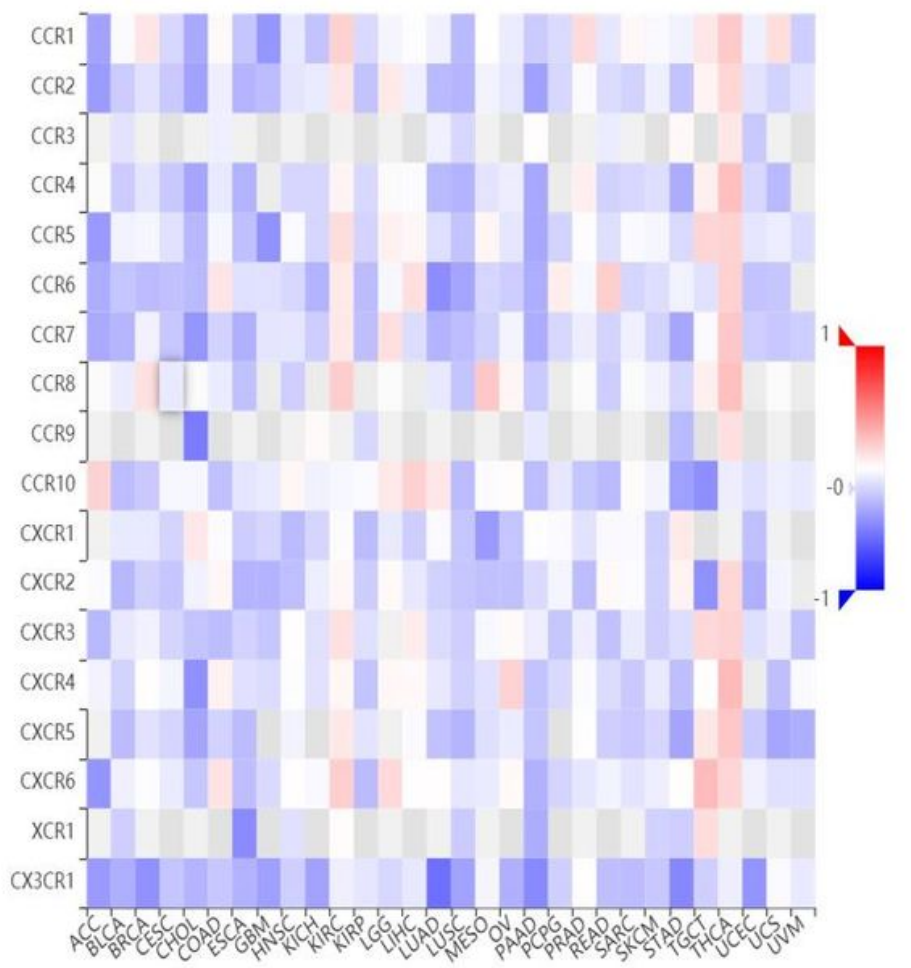

B
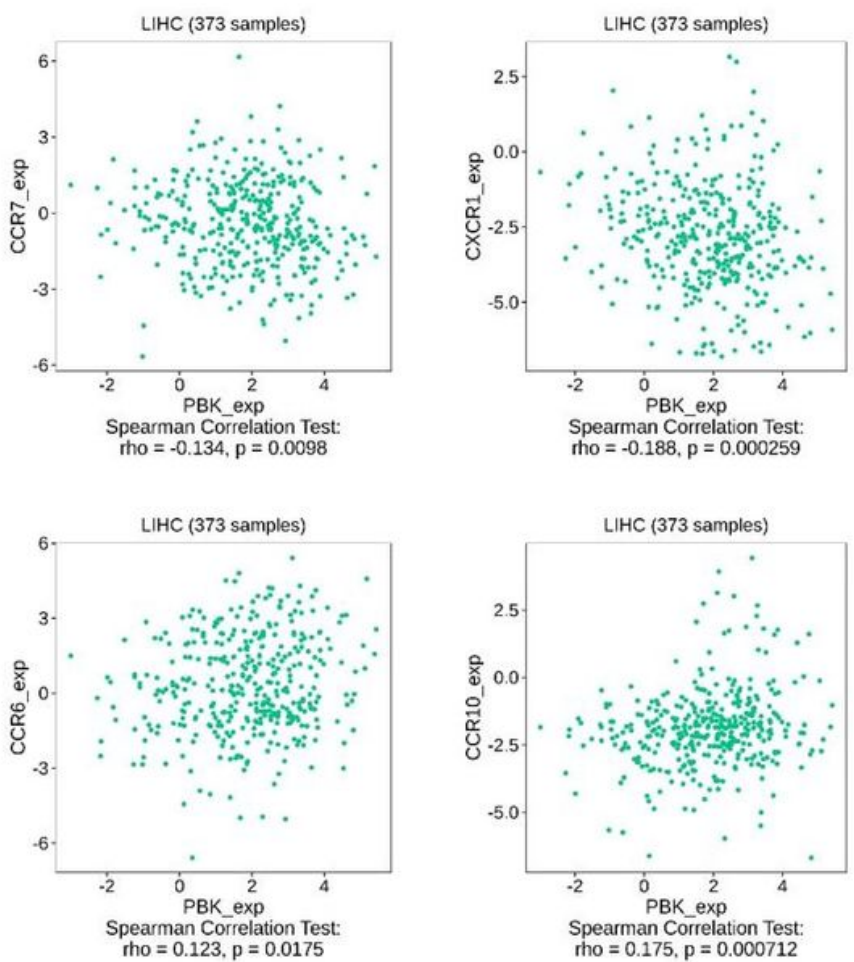

\section{Figure 10}

A: Relations between receptors and expression of PBK in pan-cancers (red is positive correlated and blue is negative correlated). B: PBK expression was positively closely related with CCR6 and CCR10, and was negatively correlated with CCR7 and CXCR1.

A
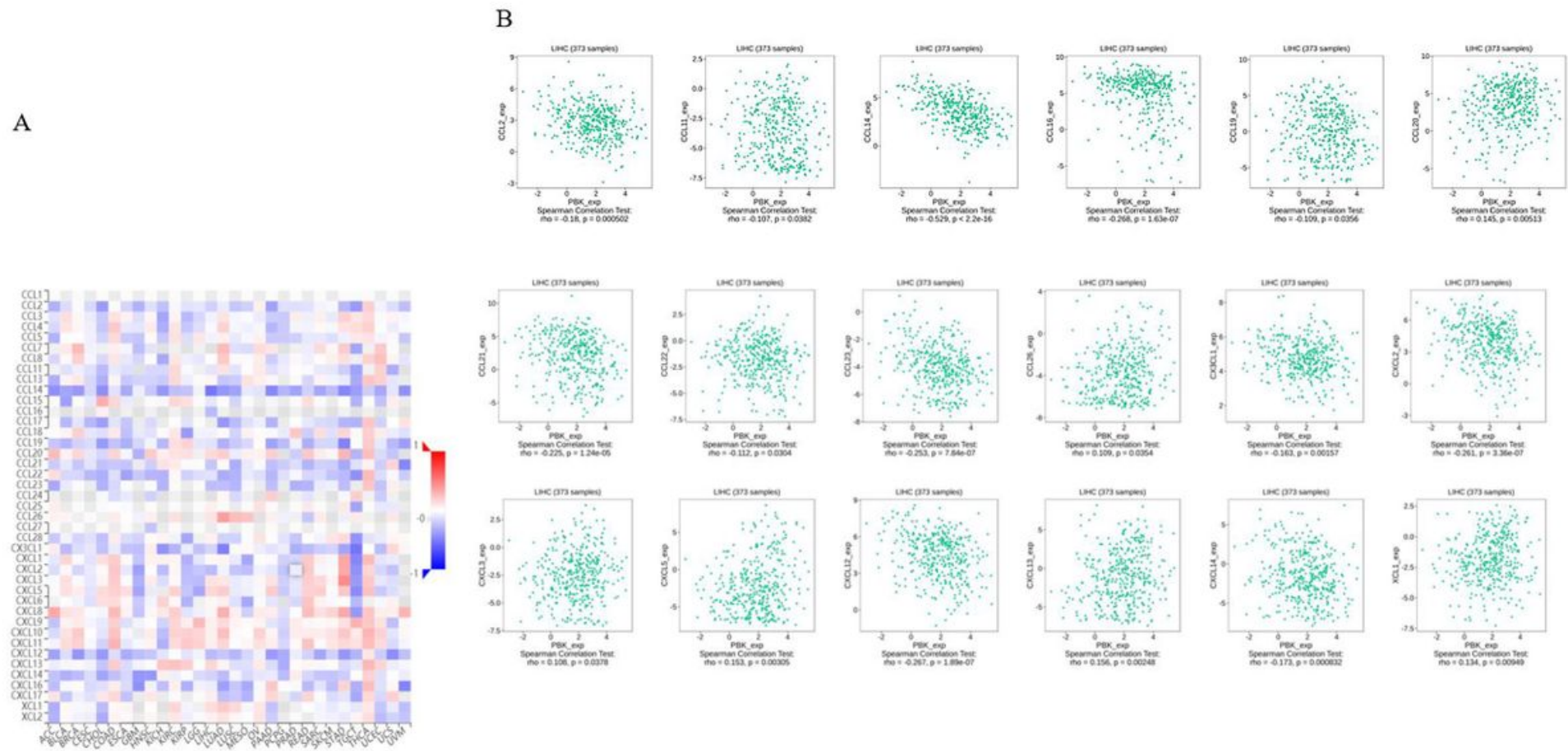

Figure 11 
A: Relations between chemokines and expression of PBK in pan-cancers (red is positive correlated and blue is negative correlated). B: PBK expression was positively or negatively closely related with chemokines (rho $>0$ indicate positively, rho<0 indicate negatively).

A
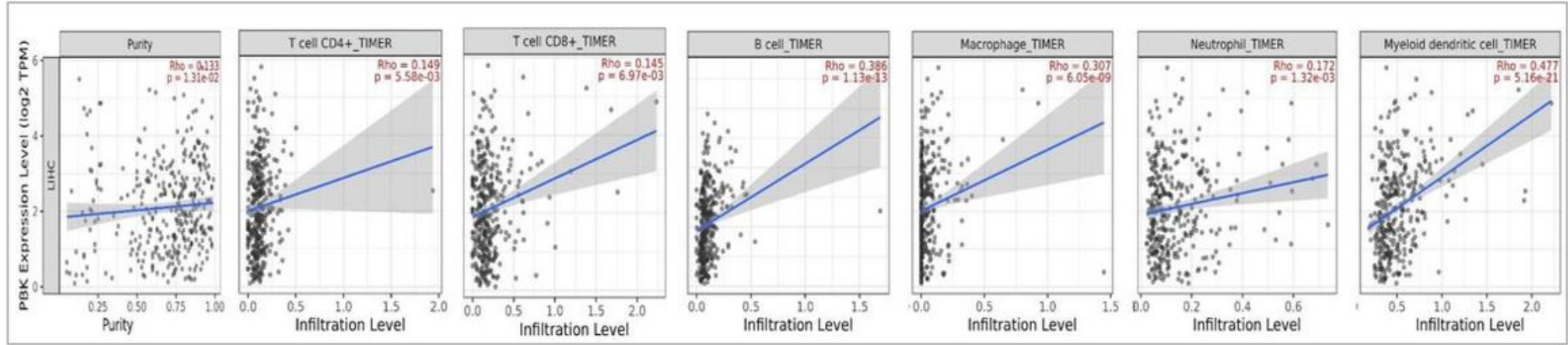

\section{$\mathrm{B}$}
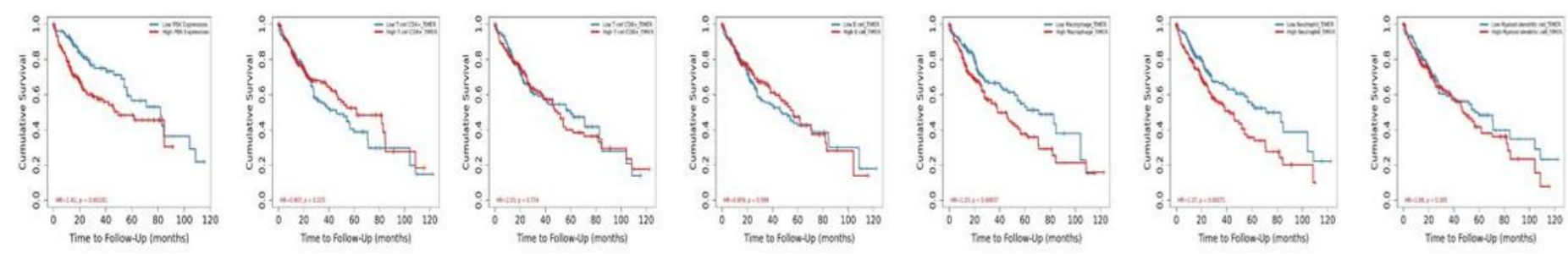

Figure 12

Correlation between PBK expression and TIICs and K-M plot of TIICs using data from TIMER. A: Correlation between TIICs and PBK expression in HCC. TIICs include B cells, CD4+ T cells, CD8+ T cells, neutrophils, macrophages, and dendritic cells. B: Kaplan-Meier survival analysis of PBK and TIICs. TIICs: tumor-infiltrating immune cells. 